\title{
Algebraic Calculation of the Energy Eigenvalues for the Nondegenerate Three-Dimensional Kepler-Coulomb Potential ${ }^{\star}$
}

\author{
Yannis TANOUDIS and Costas DASKALOYANNIS \\ Mathematics Department, Aristotle University of Thessaloniki, 54124 Greece \\ E-mail: tanoudis@math.auth.gr,daskalo@math.auth.gr
}

Received February 01, 2011, in final form May 22, 2011; Published online June 03, 2011

doi:10.3842/SIGMA.2011.054

\begin{abstract}
In the three-dimensional flat space, a classical Hamiltonian, which has five functionally independent integrals of motion, including the Hamiltonian, is characterized as superintegrable. Kalnins, Kress and Miller (J. Math. Phys. 48 (2007), 113518, 26 pages) have proved that, in the case of nondegenerate potentials, i.e. potentials depending linearly on four parameters, with quadratic symmetries, posses a sixth quadratic integral, which is linearly independent of the other integrals. The existence of this sixth integral imply that the integrals of motion form a ternary quadratic Poisson algebra with five generators. The superintegrability of the generalized Kepler-Coulomb potential that was investigated by Verrier and Evans (J. Math. Phys. 49 (2008), 022902, 8 pages) is a special case of superintegrable system, having two independent integrals of motion of fourth order among the remaining quadratic ones. The corresponding Poisson algebra of integrals is a quadratic one, having the same special form, characteristic to the nondegenerate case of systems with quadratic integrals. In this paper, the ternary quadratic associative algebra corresponding to the quantum Verrier-Evans system is discussed. The subalgebras structure, the Casimir operators and the the finite-dimensional representation of this algebra are studied and the energy eigenvalues of the nondegenerate Kepler-Coulomb are calculated.
\end{abstract}

Key words: superintegrable; quadratic algebra; Coulomb potential; Verrier-Evans potential; ternary algebra

2010 Mathematics Subject Classification: 81R12; 37J35; 70H06; 17C90

\section{Introduction}

In the $N$-dimensional space one Hamiltonian system characterized as superintegrable if it has $2 N-1$ integrals.

Kalnins, Kress and Miller have studied [1, 2] three-dimensional superintegrable systems, whose the potentials depend on four constants; these systems are referred as nondegenerate potentials. The case in which one three-dimensional potential has fewer "free parameters" than four, defines a potential, which is called degenerate potential. Verrier and Evans [3] have introduced a new classical superintegrable Hamiltonian,

$$
H=\frac{1}{2}\left(p_{x}^{2}+p_{y}^{2}+p_{z}^{2}\right)-\frac{k}{\sqrt{x^{2}+y^{2}+z^{2}}}+\frac{k_{1}}{x^{2}}+\frac{k_{2}}{y^{2}}+\frac{k_{3}}{z^{2}},
$$

which is a nondegenerate generalized Kepler-Coulomb system. The above Hamiltonian is a superintegrable system with quadratic and quartic, in momenta, integrals of motion. The quartic

\footnotetext{
${ }^{\star}$ This paper is a contribution to the Special Issue "Symmetry, Separation, Super-integrability and Special Functions $\left(\mathrm{S}^{4}\right)$ ". The full collection is available at http://www.emis.de/journals/SIGMA/S4.html
} 
integrals are generalizations of the Laplace-Runge-Lenz vectors of the ordinary Kepler-Coulomb potential.

The quantum form of the nondegenerate Kepler-Coulomb Hamiltonian is

$$
\begin{aligned}
H= & -\frac{\hbar^{2}}{2}\left(\partial_{x x}+\partial_{y y}+\partial_{z z}\right)-\frac{\hbar^{2} \mu}{\sqrt{x^{2}+y^{2}+z^{2}}} \\
& +\frac{\hbar^{2}\left(4 \mu_{1}^{2}-1\right)}{8 x^{2}}+\frac{\hbar^{2}\left(4 \mu_{2}^{2}-1\right)}{8 y^{2}}+\frac{\hbar^{2}\left(4 \mu_{3}^{2}-1\right)}{8 z^{2}} .
\end{aligned}
$$

Kalnins, Williams, Miller and Pogosyan in [4] have studied the energy eigenvalues using the method of separation of variables for potentials, one among them is the generalized KeplerCoulomb system. In this paper the energy eigenvalues of the nondegenerate generalized KeplerCoulomb system are calculated by using algebraic methods. In Section 2 we recall the method of calculation of energy eigenvalues using quadratic ternary algebras [5]. In Section 3 the structure of the algebra generated by the integrals of the nondegenerate Kepler-Coulomb system is studied. In Section 4 we apply the method of Section 2 and we calculate the energy eigenvalues of the nondegenerate Coulomb system. The commutation relations of the quadratic algebra are given in [6], for clarity reasons these relations are reproduced in the Appendix.

\section{Quadratic algebra for two-dimensional quantum superintegrable systems with quadratic integrals}

Any two-dimensional quantum superintegrable system with integrals quadratic in momenta is described by the Hamiltonian $H$ and two functionally independent integrals of motion $A$ and $B$. The integrals $A$ and $B$ commute with Hamiltonian $H$, but they don't commute between them

$$
[H, A]=0, \quad[H, B]=0 \quad \text { and } \quad[A, B] \neq 0 .
$$

The above relations can be presented by the following diagram

$$
A---H---B
$$

where the dashed line joining two operators means that the corresponding commutator is zero, the absence of any joining line between $A$ and $B$ means that the corresponding commutator is different to zero

Let $\mathcal{A}=\mathbb{C}\langle A, B, H\rangle$ be the unital algebra generated by the operators $A, B, H$, the generators of this algebra satisfy ternary relations, which are quadratic extensions of the enveloping algebra of a Lie triple system [7]

$$
\begin{aligned}
& {[A,[A, B]]=\alpha A^{2}+\beta B^{2}+\gamma\{A, B\}+\delta A+\epsilon B+\zeta,} \\
& {[B,[A, B]]=a A^{2}-\gamma B^{2}-\alpha\{A, B\}+d A-\delta B+z .}
\end{aligned}
$$

In a Lie triple system [7] in the right hand side of the above equations are only linear functions of the operators $A$ and $B$. In the case of superintegrable systems with quadratic integrals of motion there are also quadratic terms. Some of the coefficients of the ternary quadratic algebra (3) depend generally on the energy $H$

$$
\begin{aligned}
& \delta=\delta_{1} H+\delta_{0}, \quad \epsilon=\epsilon_{1} H+\epsilon_{0}, \\
& \zeta=\zeta_{2} H^{2}+\zeta_{1} H+\zeta_{0}, \quad d=d_{1} H+d_{0}, \quad z=z_{2} H^{2}+z_{1} H+z_{0} .
\end{aligned}
$$


We are interested to calculate the energy eigenvalues of the operator $H$, therefore we search to calculate the values of the energy corresponding to finite-dimensional representations of the algebra (3).

There is a Casimir $[K, A]=[K, B]=0$ and $K=K(H)$ :

$$
\begin{aligned}
K= & {[A, B]^{2}-\alpha\left\{A^{2}, B\right\}-\gamma\left\{A, B^{2}\right\}+\left(\alpha \gamma-\delta+\frac{a \beta}{3}\right)\{A, B\}-\frac{2 \beta}{3} B^{3} } \\
& +\left(\gamma^{2}-\epsilon-\frac{\alpha \beta}{3}\right) B^{2}+\left(-\gamma \delta+2 \zeta-\frac{\beta d}{3}\right) B+\frac{2 a}{3} A^{3} \\
& +\left(d+\frac{a \gamma}{3}+\alpha^{2}\right) A^{2}+\left(\frac{a \epsilon}{3}+\alpha \delta+2 z\right) A=h_{0}+h_{1} H+h_{2} H^{2}+h_{3} H^{3} .
\end{aligned}
$$

In [8] the classical two-dimensional superintegrals with quadratic integrals of motion satisfy a Poisson quadratic algebra. All the quantum superintegrable systems with quadratic integrals of motion satisfy the algebra (2)-(5) [9]. The classical three-dimensional superintegrable systems with quadratic integrals on a flat space have a structure of ternary quadratic algebra [10].

In [5] the unitary representation of this algebra is studied. In the case of $\gamma \neq 0$ and $\beta=0$, the eigenvalues of the operator $A$ are given by the formula

$$
A(x)=\frac{\gamma}{2}\left((x+u)^{2}-\frac{1}{4}-\frac{\epsilon}{\gamma^{2}}\right)
$$

where $x=0,1,2, \ldots$. The energy eigenvalues of the operator $H$ with degeneracy equal to $p+1$, where $p=0,1,2, \ldots$ are determined by solving the system of equations

$$
\Phi(0, u)=0, \quad \Phi(p+1, u)=0, \quad \text { and } \quad \Phi(x, u)>0, \quad \text { where } \quad x=1,2, \ldots, p .
$$

The structure function is given by the next relation

$$
\begin{aligned}
\Phi(x)= & -3072 K(2(u+x)-1)^{2} \gamma^{6}-48(2(u+x)-3)(2(u+x)-1)^{4} \\
& \times(2(u+x)+1) \gamma^{6}\left(\epsilon \alpha^{2}-\gamma \delta \alpha-d \gamma^{2}+a \gamma \epsilon\right)+(2(u+x)-3)^{2}(2(u+x)-1)^{4} \\
& \times(2(u+x)+1)^{2}\left(3 \alpha^{2}+4 a \gamma\right) \gamma^{8}+768\left(4 \zeta \gamma^{2}-2 \delta \epsilon \gamma+\alpha \epsilon^{2}\right)^{2}+32(2(u+x)-1)^{2} \\
& \times\left(12(u+x)^{2}-12(u+x)-1\right) \gamma^{4}\left(8 z \gamma^{3}+2 \delta^{2} \gamma^{2}-4 d \epsilon \gamma^{2}+4 \alpha \zeta \gamma^{2}+2 a \epsilon^{2} \gamma\right. \\
& \left.-6 \alpha \delta \epsilon \gamma+3 \alpha^{2} \epsilon^{2}\right)-256(2(u+x)-1)^{2} \gamma^{2}\left(-4 z \gamma^{5}+2 \delta^{2} \gamma^{4}+2 d \epsilon \gamma^{4}+4 \alpha \zeta \gamma^{4}\right. \\
& \left.+12 z \epsilon \gamma^{3}-12 \delta \zeta \gamma^{3}-3 d \epsilon^{2} \gamma^{2}+6 \delta^{2} \epsilon \gamma^{2}+12 \alpha \epsilon \zeta \gamma^{2}+a \epsilon^{3} \gamma-9 \alpha \delta \epsilon^{2} \gamma+3 \alpha^{2} \epsilon^{3}\right)
\end{aligned}
$$

In [5] this method is used for the calculation of energy eigenvalues for the two-dimensional superintegrable systems on the plane. This quadratic, cubic and generally polynomial algebras is the subject of current investigations. The cubic extension of the above algebra for superintegrable systems with an integral of motion cubic in momenta is studied in a series of papers by I. Marquette and P. Winternitz [11, 12], by I. Marquette [13, 14, 15]. The case of higher order integrals of motion can be found in [16], the case of one-dimensional position-dependent mass Schrödinger equation in [17]. These methods were applied to three-dimensional MICZ-Kepler system in [18].

\section{Quadratic algebra for the nondegenerate Kepler-Coulomb system}

The nondegenerate Kepler-Coulomb system (1) possesses three quadratic integrals which denoted by $A_{1}, A_{2}, B_{2}$ and one integral of fourth order in addition to last mentioned quadratic 
ones, which denoted by $B_{1}$

$$
\begin{aligned}
& A_{1}=\frac{1}{2} J^{2}+\frac{\hbar^{2}\left(4 \mu_{1}^{2}-1\right)\left(y^{2}+z^{2}\right)}{8 x^{2}}+\frac{\hbar^{2}\left(4 \mu_{2}^{2}-1\right)\left(x^{2}+z^{2}\right)}{8 y^{2}}+\frac{\hbar^{2}\left(4 \mu_{3}^{2}-1\right)\left(x^{2}+y^{2}\right)}{8 z^{2}}, \\
& A_{2}=\frac{1}{2} J_{3}^{2}+\frac{\hbar^{2}\left(4 \mu_{1}^{2}-1\right) y^{2}}{8 x^{2}}+\frac{\hbar^{2}\left(4 \mu_{2}^{2}-1\right) x^{2}}{8 y^{2}}, \\
& B_{2}=\frac{1}{2} J_{2}^{2}+\frac{\hbar^{2}\left(4 \mu_{1}^{2}-1\right) z^{2}}{8 x^{2}}+\frac{\hbar^{2}\left(4 \mu_{3}^{2}-1\right) x^{2}}{8 z^{2}}, \\
& p_{x}=-i \hbar \partial_{x}, \quad p_{y}=-i \hbar \partial_{y}, \quad p_{z}=-i \hbar \partial_{z}, \quad J_{1}=i \hbar\left(z \partial_{y}-y \partial_{z}\right), \\
& J_{2}=i \hbar\left(x \partial_{z}-z \partial_{x}\right), \quad J_{3}=i \hbar\left(y \partial_{x}-x \partial_{y}\right), \quad J=J_{1}^{2}+J_{2}^{2}+J_{3}^{2}, \\
& B_{1}=\left(\frac{1}{2}\left\{J_{1}, p_{y}\right\}-\frac{1}{2}\left\{J_{2}, p_{x}\right\}-2 z\left(\frac{-\hbar^{2} \mu}{2 \sqrt{x^{2}+y^{2}+z^{2}}}+\frac{\hbar^{2}\left(4 \mu_{1}^{2}-1\right)}{8 x^{2}}+\frac{\hbar^{2}\left(4 \mu_{2}^{2}-1\right)}{8 y^{2}}\right.\right. \\
& \left.\left.+\frac{\hbar^{2}\left(4 \mu_{3}^{2}-1\right)}{8 z^{2}}\right)\right)^{2}+\left\{\frac{1}{4}\left(\left\{x, p_{x}\right\}+\left\{y, p_{y}\right\}+\left\{z, p_{z}\right\}\right)^{2}, \frac{\hbar^{2}\left(4 \mu_{3}^{2}-1\right)}{8 z^{2}}\right\}+\frac{5 \hbar^{4}\left(4 \mu_{1}^{2}-1\right)}{16 z^{2}} .
\end{aligned}
$$

The above operators satisfy the following zero commutation relations

$$
\left[H, A_{i}\right]=0, \quad\left[H, B_{i}\right]=0, \quad\left[A_{1}, B_{2}\right]=0, \quad\left[A_{2}, B_{1}\right]=0 .
$$

According to Kalnins-Kress-Miller "5 to 6" theorem [1], in any three-dimensional Hamiltonian system with nondegenerate potential with quadratic integrals of motion, there is always exist a 6th integral that is functionally depended with the other integrals. This sixth integral of motion is linearly independent with the 5 functionally independent integrals. In the case of Kepler-Coulomb potential the last mentioned sixth integral is an integral of fourth order in momenta similar to the integral $B_{1}$, given by the following formula

$$
\begin{aligned}
F= & \left(-\frac{1}{2}\left\{J_{1}, p_{z}\right\}+\frac{1}{2}\left\{J_{3}, p_{x}\right\}\right. \\
& \left.-2 y\left(\frac{-\hbar^{2} \mu}{2 \sqrt{x^{2}+y^{2}+z^{2}}}+\frac{\hbar^{2}\left(4 \mu_{1}^{2}-1\right)}{8 x^{2}}+\frac{\hbar^{2}\left(4 \mu_{2}^{2}-1\right)}{8 y^{2}}+\frac{\hbar^{2}\left(4 \mu_{3}^{2}-1\right)}{8 z^{2}}\right)\right)^{2} \\
& +\left\{\frac{1}{4}\left(\left\{x, p_{x}\right\}+\left\{y, p_{y}\right\}+\left\{z, p_{z}\right\}\right)^{2}, \frac{\hbar^{2}\left(4 \mu_{2}^{2}-1\right)}{8 y^{2}}\right\}+\frac{5 \hbar^{4}\left(4 \mu_{2}^{2}-1\right)}{16 y^{2}} .
\end{aligned}
$$

This integral satisfies the following commutation relations

$$
[H, F]=0, \quad\left[F, B_{2}\right]=0 .
$$

The graph representing the zero commutation relations (7) and (8) is the following one

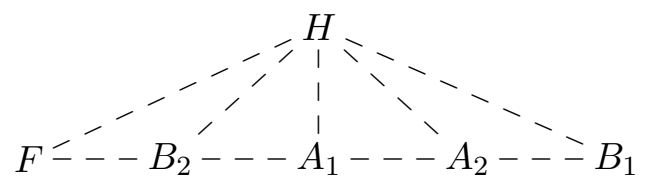

In Appendix A all the non zero ternary relations are given. By inspecting the relations (14) and (15) in the Appendix, we can see that the unital algebra generated by the operators $A_{1}$, $B_{1}, A_{2}, H$ corresponds to the graph:

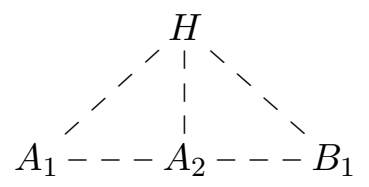


This is a quadratic subalgebra corresponding to some quadratic algebra of the form given by equations (3)-(5) but the coefficients (4) depend on the operators $\mathrm{H}$ and $A_{2}$

$$
\begin{aligned}
\alpha= & -16 \hbar^{2} H, \quad \gamma=4 \hbar^{2}, \quad \delta=16 \hbar^{2} A_{2} H-2 \hbar^{4}\left(4 \mu_{1}^{2}+4 \mu_{2}^{2}+12 \mu_{3}^{2}\right) H-4 \hbar^{6} \mu^{2}, \\
\epsilon= & 2 \hbar^{4}\left(2 \mu_{1}^{2}+2 \mu_{2}^{2}+2 \mu_{3}^{2}-3\right), \quad d=16 \hbar^{4}\left(5-4 \mu_{3}^{2}\right) H^{2}, \quad a=0, \\
z= & -32 \hbar^{4} A_{2} H^{2}+4 \hbar^{8} \mu^{2}\left(3-4 \mu_{3}^{2}\right) H \\
& +2 \hbar^{6}\left(12 \mu_{1}^{2}+12 \mu_{2}^{2}-16 \mu_{3}^{4}-8\left(2 \mu_{1}^{2}+2 \mu_{2}^{2}-3\right)-1\right) H^{2}, \\
\zeta= & 4 \hbar^{6} \mu^{2} A_{2}+2 \hbar^{6} \mu^{2}\left(1-2 \mu_{3}^{2}\right)+2\left(4 \mu_{1}^{2}+4 \mu_{2}^{2}+4 \mu_{3}^{2}\right) A_{2} H \\
& +\hbar^{6}\left(1-2 \mu_{3}^{2}\left(4 \mu_{1}^{2}+4 \mu_{2}^{2}+4 \mu_{3}^{2}-5\right)\right) H .
\end{aligned}
$$

Moreover, the above quadratic subalgebra possesses a Casimir invariant given from the following expression

$$
\begin{aligned}
K_{1}= & 4 \mu^{4}\left(\mu_{3}^{2}-1\right) \hbar^{12}+4 \hbar^{10} \mu^{2}\left(4 \mu_{3}^{4}-23 \mu_{3}^{2}+4 \mu_{1}^{2}\left(\mu_{3}^{2}-1\right)+4 \mu_{2}^{2}\left(\mu_{3}^{2}-1\right)+8\right) H \\
& +56 \hbar^{8} \mu^{2} A_{2} H+\hbar^{8}\left(16\left(\mu_{3}^{2}-1\right) \mu_{1}^{4}+4\left(8 \mu_{3}^{4}-34 \mu_{3}^{2}+8 \mu_{2}^{2}\left(\mu_{3}^{2}-1\right)+5\right) \mu_{1}^{2}+97 \mu_{3}^{2}\right. \\
& \left.+4\left(4 \mu_{3}^{6}-42 \mu_{3}^{4}+4 \mu_{2}^{4}\left(\mu_{3}^{2}-1\right)+\mu_{2}^{2}\left(8 \mu_{3}^{4}-34 \mu_{3}^{2}+5\right)\right)+15\right) H^{2} \\
& +4 \hbar^{6}\left(28 \mu_{1}^{2}+28 \mu_{2}^{2}+52 \mu_{3}^{2}-31\right) A_{2} H^{2}-48 \hbar^{4} A_{2}^{2} H^{2} .
\end{aligned}
$$

We must remark that Marquette [18] studying the MICZ-Kepler system has found quadratic algebras with coefficients, which depend of two commuting operators.

By inspecting the relations (16) and (17) in the Appendix, we can see that the unital algebra generated by the operators $A_{2}, B_{2}, A_{1}$ corresponds to the graph

$$
B_{2}---A_{1}---A_{2}
$$

This is a quadratic subalgebra corresponding to some quadratic algebra of the form given by equations (3)-(5) the coefficients (4) depend on the operator $A_{1}$

$$
\begin{aligned}
& \alpha=\gamma=4 \hbar^{2}, \quad \delta=-4 \hbar^{2} A_{1}+\hbar^{4}\left(2 \mu_{1}^{2}-3\right), \quad \epsilon=2 \hbar^{4}\left(2 \mu_{1}^{2}+2 \mu_{2}^{2}-3\right), \\
& \zeta=\hbar^{6}\left(\mu_{2}^{2}-\mu_{1}^{2}\right)+\hbar^{4}\left(3-4 \mu_{1}^{2}\right) A_{1}, \quad \beta=a=0, \quad d=-2 \hbar^{4}\left(2 \mu_{1}^{2}+2 \mu_{2}^{2}-3\right), \\
& z=\hbar^{6}\left(\mu_{1}^{2}-\mu_{3}^{2}\right)+\hbar^{4}\left(4 \mu_{1}^{2}-3\right) A_{1} .
\end{aligned}
$$

This subalgebra possesses a Casimir invariant that it is given as follows

$$
\begin{aligned}
K_{2}= & \frac{1}{8}\left(\left(-32\left(\mu_{3}^{2}-1\right) \mu_{2}^{2}+32 \mu_{3}^{2}+2\right) \mu_{1}^{2}-30 \mu_{3}^{2}+\mu_{2}^{2}\left(32 \mu_{3}^{2}-30\right)+9\right) \hbar^{8} \\
& +\frac{3}{2} \hbar^{6}\left(12 \mu_{1}^{2}-7\right) A_{1}+4 \hbar^{4}\left(\mu_{1}^{2}-1\right) A_{1}^{2} .
\end{aligned}
$$

The relations (18) and (19) in the Appendix imply that the quadratic subalgebra corresponding to the following diagram

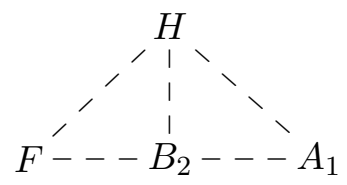

is a quadratic subalgebra of the form (3)-(5). 


\section{Calculation of the energy eigenvalues}

Using the theory given in Section 2 for the subalgebra generated by the operators $A_{2}, B_{2}, A_{1}$ (see graph (11) and the coefficients given by equation (12)), the structure function $\Phi(u, x)$ in equation (6) is written

$$
\begin{aligned}
\Phi(u, x)= & 3 \cdot 2^{18} \hbar^{16}\left(2(u+x)-\mu_{1}-\mu_{2}-1\right)\left(2(u+x)+\mu_{1}-\mu_{2}-1\right) \\
& \times\left(2(u+x)-\mu_{1}+\mu_{2}-1\right)\left(2(u+x)+\mu_{1}+\mu_{2}-1\right) \\
& \times\left(8 \hbar^{2}(u+x)^{2}-8 \hbar^{2}\left(\mu_{3}+1\right)(u+x)-2 \hbar^{2}\left(\mu_{1}^{2}+\mu_{2}^{2}-\frac{1}{2}\right)\right. \\
& \left.+4 \hbar^{2}\left(\mu_{3}+\frac{1}{2}\right)-4 A_{1}\right)\left(8 \hbar^{2}(u+x)^{2}+8 \hbar^{2}\left(\mu_{3}-1\right)(u+x)\right. \\
& \left.-2 \hbar^{2}\left(\mu_{1}^{2}+\mu_{2}^{2}-\frac{1}{2}\right)-4 \hbar^{2}\left(\mu_{3}-\frac{1}{2}\right)-4 A_{1}\right) .
\end{aligned}
$$

The value of parameter $u$ corresponding to the representation of the ternary algebra of dimension $p+1$ as well the eigenvalues of the operator $A_{1}$ determined by the next relations

$$
\Phi(u, 0)=\Phi(u, p+1)=0 .
$$

Since the structure function is a positive for $x=1,2, \ldots, p$ the values of $u$ and the corresponding eigevalues of $A_{2}$ and $A_{1}$ can be calculated analytically.

\section{Class I.}

$$
\begin{aligned}
& u=\frac{1}{2}+\frac{\mu_{1}+\mu_{2}}{2}, \\
& 2 A_{2}(x)=\hbar^{2} M^{2}-\hbar^{2}\left(\mu_{1}^{2}+\mu_{2}^{2}\right)+\frac{\hbar^{2}}{2},
\end{aligned}
$$

where $M=2 x+\mu_{1}+\mu_{2}+1, x=0,1, \ldots, p$,

$$
\begin{aligned}
\Phi(x)= & 3 \hbar^{20} 2^{28} x(p-x+1)\left(x+\mu_{1}\right)\left(x+\mu_{2}\right)\left(x+\mu_{1}+\mu_{2}\right)\left(p-x+\mu_{3}+1\right) \\
& \times\left(p+x+\mu_{1}+\mu_{2}+1\right)\left(p+x+\mu_{1}+\mu_{2}+\mu_{3}+1\right) .
\end{aligned}
$$

\section{Class II.}

$$
\begin{aligned}
& u=-\frac{1}{2}(1+2 p)-\frac{\mu_{1}+\mu_{2}}{2}, \\
& 2 A_{2}(x)=\hbar^{2} M^{2}-\hbar^{2}\left(\mu_{1}^{2}+\mu_{2}^{2}\right)+\frac{\hbar^{2}}{2},
\end{aligned}
$$

where $M=2(p-x)+\mu_{1}+\mu_{2}+1$,

$$
\begin{aligned}
& \Phi(x)=3 \hbar^{20} 2^{28} x(p-x+1)\left(p-x+\mu_{1}+1\right)\left(p-x+\mu_{2}+1\right)\left(2 p-x+\mu_{1}+\mu_{2}+2\right) \\
& \times\left(p-x+\mu_{1}+\mu_{2}+1\right)\left(x-\mu_{3}\right)\left(2 p-x+\mu_{1}+\mu_{2}+\mu_{3}+2\right) .
\end{aligned}
$$

The eigenvalues of $A_{1}$ have the form:

$$
2 A_{1}=\hbar^{2} J(J+1)-\hbar^{2}\left(\mu_{1}^{2}+\mu_{2}^{2}+\mu_{3}^{2}\right)+\frac{3}{4} \hbar^{2},
$$

where $J=2 p+\mu_{1}+\mu_{2}+\mu_{3}+\frac{3}{2}$.

The eigenvalues of the operator $A_{1}$ are given by the formula (13), where $p \geq m$. The eigenvalues are calculated using the method described in Section 2 for the subalgebra generated by the operators $A_{2}, B_{2}, A_{1}$. 
Let now consider the subalgebra generated by the operators $A_{1}, B_{1}, A_{2}, H$, see equations (9) and (10). The coefficients (10) of this algebra contain the eigenvalues of the operator $A_{2}$, which was calculated previously

$$
A_{2}=\frac{\hbar^{2}}{2}\left(2 m+\mu_{1}+\mu_{2}+1\right)^{2}-\frac{\hbar^{2}}{2}\left(\mu_{1}^{2}+\mu_{2}^{2}\right)+\frac{\hbar^{2}}{4}
$$

where $m=x$ or $m=p-x$ with $x=0, \ldots, p$ and $p \geq m$.

The eigenvalues of the of the operator $A_{1}$, using the theory of Section 2 for the subalgebra generated by the operators $A_{1}, B_{1}, A_{2}, H$ is given by the formula

$$
A_{1}(y)=\frac{\gamma}{2}\left((y+v)^{2}-\frac{1}{4}-\frac{\epsilon}{\gamma^{2}}\right)
$$

From (10) we have that

$$
\gamma=4 \hbar^{2}, \quad \epsilon=2 \hbar^{4}\left(2 \mu_{1}^{2}+2 \mu_{2}^{2}+2 \mu_{3}^{2}-3\right) .
$$

This formula should coincide with the formula calculated by equation (13), therefore

$$
y=p \quad \text { and } \quad v=\frac{1}{2}\left(2+\mu_{1}+\mu_{2}+\mu_{3}\right) .
$$

The structure function $\Phi(v, y)$ has the following form

$$
\begin{aligned}
\Phi(v, y)= & 3 \cdot 2^{18} \hbar^{16}\left(2 \hbar^{2} \mu^{2}+(4(v+y)-3)^{2} H\right)\left(2 \hbar^{2} \mu^{2}+(4(v+y)-1)^{2} H\right) \\
& \left(8 \hbar^{2}(v+y)^{2}-8 \hbar^{2}(v+y)\left(\mu_{3}+1\right)-2 \hbar^{2}\left(\mu_{1}^{2}+\mu_{2}^{2}-\mu_{3}^{2}-\frac{1}{2}\right)\right. \\
& \left.+4 \hbar^{2}\left(\mu_{3}+\frac{1}{2}\right)-4 A_{2}\right)\left(8 \hbar^{2}(v+y)^{2}+8 \hbar^{2}(v+y)\left(\mu_{3}-1\right)\right. \\
& \left.-2 \hbar^{2}\left(\mu_{1}^{2}+\mu_{2}^{2}-\mu_{3}^{2}-\frac{1}{2}\right)-4 \hbar^{2}\left(\mu_{3}-\frac{1}{2}\right)-4 A_{2}\right),
\end{aligned}
$$

where $y=m, \ldots, q$. The final form of the function $\Phi(v, y)$ with the above substitutions of $A_{2}$ and $v$ is

$$
\begin{aligned}
\Phi(v, y)= & 3 \cdot 2^{24} \hbar^{20}(y-m)\left(1+m+y+\mu_{1}+\mu_{2}\right)\left(y-m+\mu_{3}\right)\left(1+m+y+\mu_{1}+\mu_{2}+\mu_{3}\right) \\
& \times\left(2 \hbar^{2} \mu^{2}+\left(1+4 y+2 \mu_{1}+2 \mu_{2}+2 \mu_{3}\right)^{2} H\right) \\
& \times\left(2 \hbar^{2} \mu^{2}+\left(3+4 y+2 \mu_{1}+2 \mu_{2}+2 \mu_{3}\right)^{2} H\right) .
\end{aligned}
$$

The condition for calculating the energy eigenvalues is

$$
\Phi(v, m)=0, \quad \Phi(v, q+1)=0, \quad \Phi(v, y)>0 \quad \text { for } \quad y=m, m+1, \ldots, q .
$$

The energy eigenvalues are calculated using the above relations

$$
H=-\frac{\hbar^{2} \mu^{2}}{2\left(\frac{5}{2}+2 q+\mu_{1}+\mu_{2}+\mu_{3}\right)^{2}}, \quad H=-\frac{\hbar^{2} \mu^{2}}{2\left(\frac{5}{2}+2 q+1+\mu_{1}+\mu_{2}+\mu_{3}\right)^{2}},
$$

where $q=0,1, \ldots$ 


\section{Discussion}

Using pure algebraic methods of [5], we can calculate the energy eigenvalues of the nondegenerate three-dimensional Kepler-Coulomb system, which is discussed be Verrier and Evans [3].

This method can be applied to other three-dimensional nondegenerate superintegrable systems and it is the object of current investigation. The multidimensional ternary quadratic algebra is an algebra generated by the operators $S_{i}$ with $i=1,2, \ldots, n$ satisfying the relations

$$
\left[S_{i},\left[S_{j}, S_{k}\right]\right]=\sum_{r \leq s} d_{i j k}^{r s}\left\{S_{r}, S_{s}\right\}+\sum_{r} c_{i j k}^{r} S_{r}+f_{i j k}
$$

The structure constants $d_{i j k}^{r s}, c_{i j k}^{r}, f_{i j k}$ should obey to complicated restrictions, due the Jacobi kind relations for the quadratic algebra. The study of this kind of algebras, which describe many multidimensional superintegrable systems is an interesting mathematical topic, which is not yet been explored.

\section{A Appendix: Ternary quadratic algebra}

$$
\begin{aligned}
& {\left[\left[A_{1}, B_{1}\right], A_{2}\right]=\left[\left[A_{2}, B_{2}\right], A_{1}\right]=\left[\left[A_{1}, F\right], B_{2}\right]=0 \text {, }} \\
& {\left[A_{1},\left[A_{1}, B_{1}\right]\right]=-16 \hbar^{2} H A_{1}^{2}+4 \hbar^{2}\left\{A_{1}, B_{1}\right\}+\left(16 \hbar^{2} A_{2} H-2 \hbar^{4}\left(4 \mu_{1}^{2}+4 \mu_{2}^{2}+12 \mu_{3}^{2}-5\right) H\right.} \\
& \left.-4 \hbar^{6} \mu^{2}\right) A_{1}+\hbar^{4}\left(4 \mu_{1}^{2}+4 \mu_{2}^{2}+4 \mu_{3}^{2}-6\right) B_{1}+2 \hbar^{8} \mu^{2}\left(1-2 \mu_{3}^{2}\right)+4 \hbar^{6} \mu^{2} A_{2} \\
& +\hbar^{6}\left(1-2 \mu_{3}^{2}\left(4 \mu_{1}^{2}+4 \mu_{2}^{2}+4 \mu_{3}^{2}-5\right)\right) H \\
& +2 \hbar^{4}\left(4 \mu_{1}^{2}+4 \mu_{2}^{2}+4 \mu_{3}^{2}-5\right) A_{2} H, \\
& {\left[B_{1},\left[A_{1}, B_{1}\right]\right]=-4 \hbar^{2} B_{1}^{2}+16 \hbar^{2} H\left\{A_{1}, B_{1}\right\}+16 \hbar^{4}\left(5-4 \mu_{3}^{2}\right) H^{2} A_{1}} \\
& -\left(16 \hbar^{2} A_{2} H-2 \hbar^{4}\left(4 \mu_{1}^{2}+4 \mu_{2}^{2}+12 \mu_{3}^{2}-5\right) H-4 \hbar^{6} \mu^{2}\right) B_{1} \\
& -2 \hbar^{6}\left(16 \mu_{3}^{4}+8\left(2 \mu_{1}^{2}+2 \mu_{2}^{2}-3\right) \mu_{3}^{2}-12 \mu_{1}^{2}-12 \mu_{2}^{2}+1\right) H^{2} \\
& +4 \hbar^{8} \mu^{2}\left(3-4 \mu_{3}^{2}\right) H-32 \hbar^{4} A_{2} H^{2} \\
& {\left[A_{2},\left[A_{2}, B_{2}\right]\right]=4 \hbar^{2} A_{2}^{2}+4 \hbar^{2}\left\{A_{2}, B_{2}\right\}+\left(-4 \hbar^{2} A_{1}+\hbar^{4}\left(4 \mu_{1}^{2}-3\right)\right) A_{2}} \\
& +\hbar^{4}\left(\hbar^{2} \mu_{2}^{2}-\frac{1}{4}\left(\hbar^{2}+16 \mu_{1}^{2}-12\right)\right) A_{1}-\frac{\hbar^{6}}{4}\left(4 \mu_{1}^{2}-1\right) \\
& +2 \hbar^{4}\left(2 \mu_{1}^{2}+2 \mu_{2}^{2}-3\right) B_{2}, \\
& {\left[B_{2},\left[A_{2}, B_{2}\right]\right]=-4 \hbar^{2} B_{2}^{2}-4 \hbar^{2}\left\{A_{2}, B_{2}\right\}-2 \hbar^{4}\left(2 \mu_{1}^{2}+2 \mu_{3}^{2}-3\right) A_{2}} \\
& -\left(-4 \hbar^{2} A_{1}+\hbar^{4}\left(4 \mu_{1}^{2}-3\right)\right) B_{2}+\frac{1}{4} \hbar^{6}\left(4 \mu_{1}^{2}-1\right) \\
& +\frac{1}{4} \hbar^{4}\left(-4 \mu_{3}^{2} \hbar^{2}+\hbar^{2}+16 \mu_{1}^{2}-12\right) A_{1}, \\
& {\left[A_{1},\left[A_{1}, F\right]\right]=\left(16 \hbar^{2} B_{2} H-2 \hbar^{4}\left(4 \mu_{1}^{2}+12 \mu_{2}^{2}+4 \mu_{3}^{2}-5\right) H-4 \hbar^{6} \mu^{2}\right) A_{1}} \\
& -16 \hbar^{2} H A_{1}^{2}+4 \hbar^{2}\left\{A_{1}, F\right\}+\hbar^{4}\left(4 \mu_{1}^{2}+4 \mu_{2}^{2}+4 \mu_{3}^{2}-6\right) F+4 \hbar^{6} \mu^{2} B_{2} \\
& +\hbar^{6}\left(1-2 \mu_{2}^{2}\left(4 \mu_{1}^{2}+4 \mu_{2}^{2}+4 \mu_{3}^{2}-5\right)\right) H+2 \hbar^{4}\left(4 \mu_{1}^{2}+4 \mu_{2}^{2}+4 \mu_{3}^{2}-5\right) B_{2} H \\
& +2 \hbar^{8} \mu^{2}\left(1-2 \mu_{2}^{2}\right) \text {, } \\
& {\left[F,\left[A_{1}, F\right]\right]=-2 \hbar^{6}\left(4\left(4 \mu_{2}^{2}-3\right) \mu_{1}^{2}-12 \mu_{3}^{2}+8 \mu_{2}^{2}\left(2 \mu_{2}^{2}+2 \mu_{3}^{2}-3\right)+1\right) H^{2}} \\
& -\left(16 \hbar^{2} B_{2} H-2 \hbar^{4}\left(4 \mu_{1}^{2}+12 \mu_{2}^{2}+4 \mu_{3}^{2}-5\right) H-4 \hbar^{6} \mu^{2}\right) F-4 \hbar^{2} F^{2} \\
& +16 \hbar^{2} H\left\{A_{1}, F\right\}+16 \hbar^{4}\left(5-4 \mu_{2}^{2}\right) H^{2} A_{1}+4 \hbar^{8} \mu^{2}\left(3-4 \mu_{2}^{2}\right) H-32 \hbar^{4} B_{2} H^{2}, \\
& {\left[A_{1},\left[B_{1}, B_{2}\right]\right]=\left[\left[A_{1}, B_{1}\right], B_{2}\right]=\hbar^{6}\left(8 \mu_{3}^{4}+\left(8 \mu_{1}^{2}-6\right) \mu_{3}^{2}+\mu_{2}^{2}\left(8 \mu_{3}^{2}-4\right)-1\right) H} \\
& +2 \mu^{2} \hbar^{8}\left(2 \mu_{3}^{2}-1\right)+16 \hbar^{2} A_{1}^{2} H-16 \hbar^{2} A_{1} A_{2} H
\end{aligned}
$$




$$
\begin{aligned}
& +2 \hbar^{4}\left(4 \mu_{1}^{2}+4 \mu_{2}^{2}+12 \mu_{3}^{2}-5\right) A_{1} H-2 \hbar^{4}\left(4 \mu_{1}^{2}+4 \mu_{2}^{2}+4 \mu_{3}^{2}-3\right) A_{2} H \\
& +4 \hbar^{4} B_{2} H-2 \hbar^{2}\left\{A_{1}, B_{1}\right\}-4 \hbar^{6} \mu^{2} A_{2}-4 \hbar^{4}\left(\mu_{1}^{2}+\mu_{3}^{2}-1\right) B_{1} \\
& +2 \hbar^{4}\left(1-2 \mu_{3}^{2}\right) F-2 \hbar^{2}\left\{B_{1}, B_{2}\right\}-2 \hbar^{2}\left\{A_{1}, F\right\}+2 \hbar^{2}\left\{A_{2}, F\right\}+4 \mu^{2} \hbar^{6} A_{1}, \\
& {\left[\left[B_{1}, B_{2}\right], A_{2}\right]=\left[\left[A_{2}, B_{2}\right], B_{1}\right]=-4 \hbar^{6} \mu^{2} A_{1}+4 \hbar^{6} \mu^{2} A_{2}+4 \hbar^{6} \mu^{2} B_{2}+\hbar^{4} B_{1}+2 \hbar^{4} F} \\
& -16 \hbar^{2} A_{1}^{2} H+16 \hbar^{2} A_{1} A_{2} H+16 \hbar^{2} A_{1} B_{2} H \\
& -2 \hbar^{4}\left(4 \mu_{1}^{2}+4 \mu_{2}^{2}+4 \mu_{3}^{2}+1\right) A_{1} H-2 \hbar^{2}\left\{B_{1}, B_{2}\right\}+2 \hbar^{2}\left\{A_{1}, F\right\} \\
& -2 \hbar^{2}\left\{A_{2}, F\right\}-\frac{1}{2} \hbar^{6}\left(4 \mu_{1}^{2}+4 \mu_{2}^{2}+4 \mu_{3}^{2}+1\right) H-4 \hbar^{2} A_{2} B_{1}-\hbar^{8} \mu^{2} \\
& +2 \hbar^{4}\left(4 \mu_{1}^{2}+4 \mu_{2}^{2}+4 \mu_{3}^{2}-1\right) A_{2} H+2 \hbar^{4}\left(4 \mu_{1}^{2}+4 \mu_{2}^{2}+4 \mu_{3}^{2}-3\right) B_{2} H \\
& +2 \hbar^{2}\left\{A_{1}, B_{1}\right\} \\
& {\left[\left[A_{1}, F\right], A_{2}\right]=\left[\left[A_{2}, F\right], A_{1}\right]=\hbar^{6}\left(8 \mu_{2}^{4}+\left(8 \mu_{1}^{2}+8 \mu_{3}^{2}-6\right) \mu_{2}^{2}-4 \mu_{3}^{2}-1\right) H} \\
& +2 \hbar^{8} \mu^{2}\left(2 \mu_{2}^{2}-1\right)+16 \hbar^{2} A_{1}^{2} H-16 \hbar^{2} A_{1} B_{2} H \\
& +2 \hbar^{4}\left(4 \mu_{1}^{2}+12 \mu_{2}^{2}+4 \mu_{3}^{2}-5\right) A_{1} H+4 \hbar^{4} A_{2} H \\
& -2 \hbar^{4}\left(4 \mu_{1}^{2}+4 \mu_{2}^{2}+4 \mu_{3}^{2}-3\right) B_{2} H-2 \hbar^{2}\left\{A_{1}, B_{1}\right\}+2 \hbar^{2}\left\{B_{1}, B_{2}\right\} \\
& -2 \hbar^{2}\left\{A_{1}, F\right\}+4 \hbar^{6} \mu^{2} A_{1}+2 \hbar^{4}\left(1-2 \mu_{2}^{2}\right) B_{1}-4 \hbar^{6} \mu^{2} B_{2} \\
& -4 \hbar^{4}\left(\mu_{1}^{2}+\mu_{2}^{2}-1\right) F-2 \hbar^{2}\left\{A_{2}, F\right\}, \\
& {\left[\left[A_{2}, F\right], B_{1}\right]=\left[\left[B_{1}, F\right], A_{2}\right]=64 \hbar^{2} A_{1}^{2} H^{2}-64 \hbar^{2} A_{1} A_{2} H^{2}-64 \hbar^{2} A_{1} B_{2} H^{2}-8 \hbar^{2}\left\{A_{1}, B_{1}\right\} H} \\
& +16 \hbar^{2} A_{2} B_{1} H+8 \hbar^{2}\left\{B_{1}, B_{2}\right\} H-8 \hbar^{2}\left\{A_{1}, F\right\} H+8 \hbar^{2}\left\{A_{2}, F\right\} H \\
& +8 \hbar^{4}\left(4 \mu_{1}^{2}+4 \mu_{2}^{2}+4 \mu_{3}^{2}+1\right) A_{1} H^{2}-8 \hbar^{4}\left(4 \mu_{1}^{2}+4 \mu_{2}^{2}+4 \mu_{3}^{2}-1\right) A_{2} H^{2} \\
& -8 \hbar^{4}\left(4 \mu_{1}^{2}+4 \mu_{2}^{2}+4 \mu_{3}^{2}-3\right) B_{2} H^{2}+16 \hbar^{6} \mu^{2} A_{1} H-16 \hbar^{6} \mu^{2} A_{2} H \\
& -16 \hbar^{6} \mu^{2} B_{2} H-8 \hbar^{4} F H-4 \hbar^{4} B_{1} H+2 \hbar^{6}\left(4 \mu_{1}^{2}+4 \mu_{2}^{2}+4 \mu_{3}^{2}+1\right) H^{2} \\
& +4 \hbar^{8} \mu^{2} H \\
& {\left[\left[A_{2}, B_{2}\right], F\right]=\left[\left[A_{2}, F\right], B_{2}\right]=16 \hbar^{2} A_{1}^{2} H-16 \hbar^{2} A_{1} A_{2} H-16 \hbar^{2} A_{1} B_{2} H} \\
& +2 \hbar^{4}\left(4 \mu_{1}^{2}+4 \mu_{2}^{2}+4 \mu_{3}^{2}+1\right) A_{1} H-2 \hbar^{4}\left(4 \mu_{1}^{2}+4 \mu_{2}^{2}+4 \mu_{3}^{2}-3\right) A_{2} H \\
& -2 \hbar^{4}\left(4 \mu_{1}^{2}+4 \mu_{2}^{2}+4 \mu_{3}^{2}-1\right) B_{2} H-2 \hbar^{2}\left\{A_{1}, B_{1}\right\}+2 \hbar^{2}\left\{B_{1}, B_{2}\right\} \\
& -2 \hbar^{2}\left\{A_{1}, F\right\}+2 \hbar^{2}\left\{A_{2}, F\right\}+4 \hbar^{2} F B_{2}+4 \hbar^{6} \mu^{2} A_{1}-4 \hbar^{6} \mu^{2} A_{2}-4 \hbar^{6} \mu^{2} B_{2} \\
& -2 \hbar^{4} B_{1}-\hbar^{4} F+\frac{1}{2} \hbar^{6}\left(4 \mu_{1}^{2}+4 \mu_{2}^{2}+4 \mu_{3}^{2}+1\right) H+\hbar^{8} \mu^{2} \\
& {\left[\left[B_{1}, B_{2}\right], F\right]=\left[\left[B_{1}, F\right], B_{2}\right]=-64 \hbar^{2} A_{1}^{2} H^{2}+64 \hbar^{2} A_{1} A_{2} H^{2}+64 \hbar^{2} A_{1} B_{2} H^{2}} \\
& +8 \hbar^{2}\left\{A_{1}, B_{1}\right\} H+8 \hbar^{2}\left\{A_{1}, F\right\} H-8 \hbar^{2}\left\{A_{2}, F\right\} H-8 \hbar^{2}\left\{B_{1}, B_{2}\right\} H \\
& -16 \hbar^{2} B_{2} F H-8 \hbar^{4}\left(4 \mu_{1}^{2}+4 \mu_{2}^{2}+4 \mu_{3}^{2}+1\right) A_{1} H^{2} \\
& +8 \hbar^{4}\left(4 \mu_{1}^{2}+4 \mu_{2}^{2}+4 \mu_{3}^{2}-3\right) A_{2} H^{2}+8 \hbar^{4}\left(4 \mu_{1}^{2}+4 \mu_{2}^{2}+4 \mu_{3}^{2}-1\right) B_{2} H^{2} \\
& -16 \hbar^{6} \mu^{2} A_{1} H+16 \hbar^{6} \mu^{2} A_{2} H+16 \hbar^{6} \mu^{2} B_{2} H+4 \hbar^{4} F H+8 \hbar^{4} B_{1} H \\
& -2 \hbar^{6}\left(4 \mu_{1}^{2}+4 \mu_{2}^{2}+4 \mu_{3}^{2}+1\right) H^{2}-4 \hbar^{8} \mu^{2} H, \\
& {\left[\left[B_{1}, F\right], A_{1}\right]=8 \hbar^{2}\left\{A_{1}, B_{1}\right\} H-8 \hbar^{2}\left\{B_{1}, B_{2}\right\} H+8 \hbar^{2}\left\{A_{2}, F\right\} H-8 \hbar^{2}\left\{A_{1}, F\right\} H} \\
& -16 \hbar^{4} A_{2} H^{2}+16 \hbar^{4} B_{2} H^{2}+8 \hbar^{4}\left(1-2 \mu_{3}^{2}\right) F H+8 \hbar^{4}\left(2 \mu_{2}^{2}-1\right) B_{1} H \\
& +16 \hbar^{6}\left(\mu_{3}^{2}-\mu_{2}^{2}\right) H^{2} \\
& {\left[\left[A_{1}, B_{1}\right], F\right]=64 \hbar^{2} A_{1}^{2} H^{2}-64 \hbar^{2} A_{1} A_{2} H^{2}-64 \hbar^{2} A_{1} B_{2} H^{2}+8 \hbar^{2}\left\{B_{1}, B_{2}\right\} H} \\
& -8 \hbar^{2}\left\{A_{1}, B_{1}\right\} H+8 \hbar^{4}\left(4 \mu_{1}^{2}+4 \mu_{2}^{2}+4 \mu_{3}^{2}+5\right) A_{1} H^{2}
\end{aligned}
$$


$-8 \hbar^{4}\left(4 \mu_{1}^{2}+4 \mu_{2}^{2}+4 \mu_{3}^{2}+1\right) A_{2} H^{2}-8 \hbar^{4}\left(4 \mu_{1}^{2}+4 \mu_{2}^{2}+4 \mu_{3}^{2}+3\right) B_{2} H^{2}$

$+4 \hbar^{4}\left(1-4 \mu_{2}^{2}\right) B_{1} H+16 \hbar^{6} \mu^{2} A_{1} H-16 \hbar^{6} \mu^{2} A_{2} H-16 \hbar^{6} \mu^{2} B_{2} H$

$-4 \hbar^{4} F H+2 \hbar^{2}\left\{B_{1}, F\right\}+2 \hbar^{6}\left(4 \mu_{1}^{2}+12 \mu_{2}^{2}+4 \mu_{3}^{2}-1\right) H^{2}+4 \hbar^{8} \mu^{2} H$,

$\left[\left[A_{1}, F\right], B_{1}\right]=64 \hbar^{2} A_{1}^{2} H^{2}-64 \hbar^{2} A_{1} A_{2} H^{2}-64 \hbar^{2} A_{1} B_{2} H^{2}$

$+8 \hbar^{4}\left(4 \mu_{1}^{2}+4 \mu_{2}^{2}+4 \mu_{3}^{2}+5\right) A_{1} H^{2}-8 \hbar^{4}\left(4 \mu_{1}^{2}+4 \mu_{2}^{2}+4 \mu_{3}^{2}+3\right) A_{2} H^{2}$

$-8 \hbar^{4}\left(4 \mu_{1}^{2}+4 \mu_{2}^{2}+4 \mu_{3}^{2}+1\right) B_{2} H^{2}-8 \hbar^{2}\left\{A_{1}, F\right\} H+8 \hbar^{2}\left\{A_{2}, F\right\} H$

$+16 \hbar^{6} \mu^{2} A_{1} H-16 \hbar^{6} \mu^{2} A_{2} H-16 \hbar^{6} \mu^{2} B_{2} H+4 \hbar^{4}\left(1-4 \mu_{3}^{2}\right) F H$

$-4 \hbar^{4} B_{1} H+2 \hbar^{2}\left\{B_{1}, F\right\}+2 \hbar^{6}\left(4 \mu_{1}^{2}+4 \mu_{2}^{2}+12 \mu_{3}^{2}-1\right) H^{2}+4 \hbar^{8} \mu^{2} H$,

$\left[\left[B_{1}, F\right], F\right]=64 \hbar^{2} B_{2} F H^{2}-32 \hbar^{2}\left\{A_{1}, F\right\} H^{2}+32 \hbar^{2}\left\{A_{2}, F\right\} H^{2}-128 \hbar^{4} A_{1} H^{3}$

$+128 \hbar^{4} A_{2} H^{3}+128 \hbar^{4} B_{2} H^{3}-16 \hbar^{4}\left(3-4 \mu_{2}^{2}\right) B_{1} H^{2}-8 \hbar^{2}\left\{B_{1}, F\right\} H$

$+16 \hbar^{6}\left(1-4 \mu_{2}^{2}\right) H^{3}$,

$\left[\left[B_{1}, F\right], B_{1}\right]=32 \hbar^{2}\left\{A_{1}, B_{1}\right\} H^{2}-64 \hbar^{2} B_{1} A_{2} H^{2}-32 \hbar^{2}\left\{B_{1}, B_{2}\right\} H^{2}+128 \hbar^{4} A_{1} H^{3}$

$-128 \hbar^{4} A_{2} H^{3}-128 \hbar^{4} B_{2} H^{3}+16 \hbar^{4}\left(3-4 \mu_{3}^{2}\right) F H^{2}+8 \hbar^{2}\left\{B_{1}, F\right\} H$

$-16 \hbar^{6}\left(1-4 \mu_{3}^{2}\right) H^{3}$,

$\left[\left[A_{2}, F\right], F\right]=-8 \hbar^{2}\left\{A_{1}, F\right\} H-8 \hbar^{2}\left\{A_{2}, F\right\} H+16 \hbar^{4}\left(4 \mu_{2}^{2}-3\right) A_{1} H^{2}-32 \hbar^{4} A_{2} H^{2}$

$+2 \hbar^{6}\left(16 \mu_{2}^{4}+16\left(\mu_{3}^{2}-1\right) \mu_{2}^{2}-12 \mu_{3}^{2}+4 \mu_{1}^{2}\left(4 \mu_{2}^{2}-3\right)-1\right) H^{2}+2 \hbar^{2}\left\{B_{1}, F\right\}$

$+4 \hbar^{2} F^{2}-2 \hbar^{4}\left(4 \mu_{1}^{2}+12 \mu_{2}^{2}+4 \mu_{3}^{2}-5\right) F H+4 \hbar^{4}\left(3-4 \mu_{2}^{2}\right) B_{1} H-4 \hbar^{6} \mu^{2} F$

$+4 \hbar^{8} \mu^{2}\left(4 \mu_{2}^{2}-3\right) H$,

$\left[\left[F, A_{2}\right], A_{2}\right]=-\frac{1}{2} \hbar^{6}\left(16 \mu_{2}^{4}+16\left(\mu_{3}^{2}-1\right) \mu_{2}^{2}+4 \mu_{1}^{2}\left(4 \mu_{2}^{2}-1\right)-3\left(4 \mu_{3}^{2}+1\right)\right) H$

$-16 \hbar^{2} A_{1} A_{2} H+4 \hbar^{2} A_{2} B_{1}+4 \hbar^{2}\left\{A_{2}, F\right\}+4 \hbar^{4}\left(3-4 \mu_{2}^{2}\right) A_{1} H$

$-2 \hbar^{4}\left(4 \mu_{1}^{2}+4 \mu_{2}^{2}+4 \mu_{3}^{2}+1\right) A_{2} H+2 \hbar^{4}\left(2 \mu_{1}^{2}+2 \mu_{2}^{2}-3\right) F$

$+\hbar^{4}\left(4 \mu_{2}^{2}-3\right) B_{1}-4 \hbar^{6} \mu^{2} A_{2}+\hbar^{8} \mu^{2}\left(3-4 \mu_{2}^{2}\right)$,

$\left[\left[B_{2}, B_{1}\right], B_{1}\right]=2 \hbar^{6}\left(16 \mu_{3}^{4}-16 \mu_{3}^{2}+4 \mu_{1}^{2}\left(4 \mu_{3}^{2}-3\right)+4 \mu_{2}^{2}\left(4 \mu_{3}^{2}-3\right)-1\right) H^{2}-32 \hbar^{4} B_{2} H^{2}$

$+2 \hbar^{2}\left\{B_{1}, F\right\}-2 \hbar^{4}\left(4 \mu_{1}^{2}+4 \mu_{2}^{2}+12 \mu_{3}^{2}-5\right) B_{1} H+4 \hbar^{2} B_{1}^{2}$

$+4 \hbar^{4}\left(3-4 \mu_{3}^{2}\right) F H-4 \hbar^{6} \mu^{2} B_{1}+4 \mu^{2} \hbar^{8}\left(4 \mu_{3}^{2}-3\right) H-8 \hbar^{2}\left\{A_{1}, B_{1}\right\} H$

$-8 \hbar^{2}\left\{B_{1}, B_{2}\right\} H+16 \hbar^{4}\left(4 \mu_{3}^{2}-3\right) A_{1} H^{2}$,

$\left[\left[B_{2}, B_{1}\right], B_{2}\right]=\frac{1}{2} \hbar^{6}\left(16 \mu_{3}^{4}-16 \mu_{3}^{2}+4 \mu_{2}^{2}\left(4 \mu_{3}^{2}-3\right)+4 \mu_{1}^{2}\left(4 \mu_{3}^{2}-1\right)-3\right) H+16 \hbar^{2} A_{1} B_{2} H$

$-4 \hbar^{2}\left\{B_{1}, B_{2}\right\}+4 \hbar^{4}\left(4 \mu_{3}^{2}-3\right) A_{1} H+2 \hbar^{4}\left(4 \mu_{1}^{2}+4 \mu_{2}^{2}+4 \mu_{3}^{2}+1\right) B_{2} H$

$-2 \hbar^{4}\left(2 \mu_{1}^{2}+2 \mu_{3}^{2}-3\right) B_{1}-4 \hbar^{2} B_{2} F+\hbar^{4}\left(3-4 \mu_{3}^{2}\right) F+4 \hbar^{6} \mu^{2} B_{2}$

$+\mu^{2} \hbar^{8}\left(4 \mu_{3}^{2}-3\right)$,

$\left[A_{1},\left[A_{1}, F\right]\right]=\left(16 \hbar^{2} B_{2} H-2 \hbar^{4}\left(4 \mu_{1}^{2}+12 \mu_{2}^{2}+4 \mu_{3}^{2}-5\right) H-4 \hbar^{6} \mu^{2}\right) A_{1}-16 \hbar^{2} H A_{1}^{2}$

$+4 \hbar^{2}\left\{A_{1}, F\right\}+\hbar^{4}\left(4 \mu_{1}^{2}+4 \mu_{2}^{2}+4 \mu_{3}^{2}-6\right) F+4 \hbar^{6} \mu^{2} B_{2}$

$+\hbar^{6}\left(1-2 \mu_{2}^{2}\left(4 \mu_{1}^{2}+4 \mu_{2}^{2}+4 \mu_{3}^{2}-5\right)\right) H+2 \hbar^{4}\left(4 \mu_{1}^{2}+4 \mu_{2}^{2}+4 \mu_{3}^{2}-5\right) B_{2} H$

$+2 \hbar^{8} \mu^{2}\left(1-2 \mu_{2}^{2}\right)$,

$\left[F,\left[A_{1}, F\right]\right]=-2 \hbar^{6}\left(4\left(4 \mu_{2}^{2}-3\right) \mu_{1}^{2}-12 \mu_{3}^{2}+8 \mu_{2}^{2}\left(2 \mu_{2}^{2}+2 \mu_{3}^{2}-3\right)+1\right) H^{2}$

$-\left(16 \hbar^{2} B_{2} H-2 \hbar^{4}\left(4 \mu_{1}^{2}+12 \mu_{2}^{2}+4 \mu_{3}^{2}-5\right) H-4 \hbar^{6} \mu^{2}\right) F-4 \hbar^{2} F^{2}$

$+16 \hbar^{2} H\left\{A_{1}, F\right\}+16 \hbar^{4}\left(5-4 \mu_{2}^{2}\right) H^{2} A_{1}+4 \hbar^{8} \mu^{2}\left(3-4 \mu_{2}^{2}\right) H-32 \hbar^{4} B_{2} H^{2}$. 


\section{References}

[1] Kalnins E.G., Kress J.M., Miller W. Jr., Nondegenerate three-dimensional complex Euclidean superintegrable systems and algebraic varieties, J. Math. Phys. 48 (2007), 113518, 26 pages, arXiv:0708.3044.

[2] Kalnins E.G., Kress J.M., Miller W. Jr., Fine structure for 3D second-order superintegrable systems: threeparameter potentials, J. Phys. A: Math. Theor. 40 (2007), 5875-5892.

[3] Verrier P.E., Evans N.W., A new superintegrable Hamiltonian, J. Math. Phys. 49 (2008), 022902, 8 pages, arXiv:0712.3677.

[4] Kalnins E.G., Williams G.C., Miller W. Jr., Pogosyan G.S., Superintegrability in three-dimensional Euclidean space, J. Math. Phys. 40 (1999), 708-725.

[5] Daskaloyannis C., Quadratic Poisson algebras of two-dimensional classical superintegrable systems and quadratic associative algebras of quantum superintegrable systems, J. Math. Phys. 42 (2001), 1100-1119, math-ph/0003017.

[6] Tanoudis Y., Daskaloyannis C., The algebra of the quantum nondegenerate three-dimensional KeplerCoulomb potential, in Proceedings of the XIIIth Conference "Symmetries in Physics" (in Memory of Professor Yurii Fedorovich Smirnov) (July 2009, Dubna), to appear.

[7] Jacobson N., General representation theory of Jordan algebras, Trans. Amer. Math. Soc. 70 (1951), 509530.

Lister W.G., A structure theory of Lie triple systems, Trans. Amer. Math. Soc. 72 (1952), 217-242.

[8] Daskaloyannis C., Ypsilantis K., Unified treatment and classification of superintegrable systems with integrals quadratic in momenta on a two dimensional manifold, J. Math. Phys. 47 (2006), 042904, 38 pages, math-ph/0412055.

[9] Daskaloyannis C., Ypsilantis K., Quantum superintegrable systems with quadratic integrals on a two dimensional manifold, J. Math. Phys. 48 (2007), 072108, 22 pages, math-ph/0607058.

[10] Tanoudis Y., Daskaloyannis C., Quadratic algebras for three-dimensional nondegenerate superintegrable systems with quadratic integrals of motion, Contribution at the XXVII Colloquium on Group Theoretical Methods in Physics (August 2008, Yerevan, Armenia), arXiv:0902.0130.

Daskaloyannis C., Tanoudis Y., Quadratic algebras for three-dimensional superintegrable systems, Phys. Atomic Nuclei 73 (2010), 214-221.

[11] Marquette I., Winternitz P., Polynomial Poisson algebras for classical superintegrable systems with a thirdorder integral of motion, J. Math. Phys. 48 (2007), 012902, 16 pages, Erratum, J. Math. Phys. 49 (2008), 019901, math-ph/0608021.

[12] Marquette I., Winternitz P., Superintegrable systems with third-order integrals of motion, J. Phys. A: Math. Theor. 41 (2008), 304031, 10 pages, arXiv:0711.4783.

[13] Marquette I., Superintegrability with third order integrals of motion, cubic algebras, and supersymmetric quantum mechanics. I. Rational function potentials, J. Math. Phys. 50 (2009), 012101, 23 pages, arXiv:0807.2858.

[14] Marquette I., Superintegrability with third order integrals of motion, cubic algebras, and supersymmetric quantum mechanics. II. Painlevé transcendent potentials, J. Math. Phys. 50 (2009), 095202, 18 pages, arXiv:0811.1568.

[15] Marquette I., Supersymmetry as a method of obtaining new superintegrable systems with higher order integrals of motion, J. Math. Phys. 50 (2009), 122102, 10 pages, arXiv:0908.1246.

[16] Marquette I., Superintegrability and higher order polynomial algebras, J. Phys. A: Math. Gen. 43 (2010), 135203, 15 pages, arXiv:0908.4399.

[17] Quesne C., Quadratic algebra approach to an exactly solvable position-dependent mass Schrödinger equation in two dimensions, SIGMA 3 (2007), 067, 14 pages, arXiv:0705.2577.

[18] Marquette I., Generalized MICZ-Kepler system, duality, polynomial, and deformed oscillator algebras, J. Math. Phys. 51 (2010), 102105, 10 pages, arXiv:1004.4579. 